\title{
Uma proposta de avaliação integrada de risco do uso de agrotóxicos no estado do Amazonas, Brasil
}

\author{
Andréa Viviana WAICHMAN ${ }^{1}$
}

\section{RESUMO}

Durante os últimos 30 anos, o aumento da população humana no Amazonas introduziu a necessidade de produção adicional de alimentos e levou o governo do estado a desenvolver programas para aumentar e melhorar a produção agrícola. A produção de hortaliças não tradicionais apresentou vários problemas desconhecidos para os agricultores da regiāo, uma vez que estas culturas não são bem adaptadas às condições locais. A suscetibilidade a insetos, fungos e outras pragas, e a competição com vegetação nativa vem forçando os agricultores a usar intensivamente os agrotóxicos. Os agricultores não estavam preparados para o uso adequado desta tecnologia ignorando os riscos dos agrotóxicos para saúde humana e o ambiente. Os agricultores não usam equipamento de proteção individual, porque é caro, desconfortável e inadequado para o clima quente da regiāo. A falta treinamento e o escasso conhecimento sobre os perigos dos agrotóxicos contribuem para a manipulação incorreta durante a preparação, aplicação e disposição das embalagens vazias. Nestas condiçôes, a exposição dos agricultores, suas famílias, consumidores e ambiente é alta. Como um primeiro passo para o entendimento deste problema e a proposição de soluções é proposta a realização de uma avaliação integrada de risco. As três fases do processo, formulação de problema, avaliação da exposição, e caracterização de risco são detalhadas. Programas de educação, treinamento e informação fazem parte das estratégias para a redução do risco do uso de agrotóxicos permitindo o desenvolvimento de uma agricultura ambientalmente sustentável.

PALAVRAS-CHAVE: Agrotóxicos, Avaliação integrada de risco, Amazônia.

\section{A proposal for integrated risk assessment of pesticide use in Amazon State, Brasil}

\begin{abstract}
During the last 30 years, the increase of the human population in the Amazon introduced the need for additional food production and caused the state government to implement programs to increase and improve agricultural production. The production of nontraditional crops introduced several problems unknown to traditional farmers, since they are not well adapted to tropical conditions. Their susceptibility to insects, fungi and other plagues, and the competition with native vegetation forced farmers to use pesticides intensively. Amazonian farmers were not adequately prepared for the use of this new technology and they ignored the risk of pesticides to human health and the environment. Farmers do not use protective clothing or equipment because they are expensive and not suitable for a tropical climate in which they prove to be very uncomfortable. The lack in training and poor knowledge of pesticide hazards contribute to careless handling during preparation, application and disposal of empty packages. Under such circumstances, exposure of farmers, their families, consumers and the environment is high. As a first step to solve this problem, an integrated risk assessment is proposed. The three stages of the process, problem formulation, exposure assessment, and risk characterization are explained. Training and information programs are part of the strategies risk-reduction in order to build up environmentally sustainable agriculture.
\end{abstract}

KEYWORDS: Pesticides, Integrated Risk Assessment, Amazon. 


\section{INTRODUÇÃO}

\section{A FORMULAÇÃO DO PROBLEMA}

Durante os últimos 30 anos a Amazônia foi objeto de uma política de desenvolvimento para o uso econômico da região que motivou assentamentos humanos principalmente associados à industria e à agricultura. A criação da Zona Franca de Manaus em 1967, originou um grande fluxo migratório para a área urbana. $\mathrm{O}$ aumento de população urbana acrescentou o problema de produção de alimentos e o governo de Estado induziu o desenvolvimento de programas para aumentar e melhorar produção agrícola, reduzir importação de comida de outras partes do país, regular o abastecimento, diminuir preços e criar condições para a fixação do homem em áreas rurais do estado. Estes programas foram principalmente desenvolvidos em áreas de várzea perto de Manaus.

A produção de hortaliças teve um grande incentivo devido principalmente à decadência da produção de juta e malva por causa da queda dos preços nos mercados nacionais e internacionais e o rápido retorno financeiro desta atividade, que é alto quando comparado com as culturas tradicionais e outras atividades econômicas desenvolvidas pelos ribeirinhos. A produção de hortaliças na várzea apresentou vários problemas para os agricultores (Noda et al., 1997). Como estes cultivos não estão adaptados às condições tropicais, a suscetibilidade ao ataque de pragas (insetos, fungos e outros) e a competição com vegetação nativa vem forçando os agricultores ao uso intensivo de agrotóxicos. Desta forma, um incremento na produtividade agrícola não pode ser alcançado sem o uso crescente de agrotóxicos, principalmente inseticidas, herbicidas e fungicidas, agentes estes que não são utilizados nas práticas agrícolas tradicionais (Ecobichon, 2001).

No estado do Amazonas a proporção de agricultores dos diferentes municípios do estado que cultivam frutas e legumes com uso de agrotóxico varia entre 64\% e 96,7\% (IBGE, 1998). Os agricultores da região não estão preparados para o uso adequado desta "tecnologia", ignoram o risco do uso de agrotóxicos para saúde e o ambiente e não recebem ajuda técnica de serviços de extensão oficiais (Waichman et al., 2002, 2003, 2007).

A quantidade de dados quantitativos e qualitativos sobre uso de agrotóxicos no Estado do Amazonas é escassa. Dados da Associação Nacional de Defesa Vegetal (ANDEF) indicam um aumento do uso de inseticidas, herbicidas e fungicidas de duas toneladas para seis toneladas de ingredientes ativos entre 1998 e 2000.

Em visitas às lojas de produtos agrícolas em Manaus, Iranduba e Manacapuru, verificamos a disponibilidade de 42 ingredientes ativos e 82 produtos comerciais, entre fungicidas, herbicidas e inseticidas (Waichman et al., 2002, 2003), alguns dos quais já foram proibidos em países desenvolvidos.
Agrotóxicos altamente perigosos ou tóxicos são livremente vendidos e usados.

A situação de analfabetismo ou baixa instrução escolar predominante entre os agricultores torna as informaçōes contidas nas etiquetas de produto difícil de entender contribuindo para o escasso conhecimento sobre a periculosidade e toxicidade do produto. Vários fatores contribuem para o uso incorreto de agrotóxicos expondo os agricultores, consumidores e o ambiente ao envenenamento (Nina, 2002; Waichman et al., 2002; 2003). Os agricultores não usam roupas ou equipamento de proteção individual (EPI) porque eles são caros e inadequados para o clima tropical. A falta de treinamento e de conhecimento dos perigos dos pesticidas contribui para uma manipulação descuidada durante preparação, aplicação e disposição das embalagens vazias, a qual a maioria das vezes é realizada nos corpos d'água próximos das áreas de plantio ou na floresta.

Os agricultores não respeitam os prazos de carência na aplicação dos agrotóxicos, borrifando as frutas e verduras até o momento de serem comercializadas (Nina, 2002; Waichman et al., 2002, 2007). Nestas circunstâncias a exposição ocupacional dos agricultores é alta, tornando-os vulneráveis à intoxicação aguda e crônica, além do risco de contaminação crônica da população consumidora de frutas e hortaliças com resíduos de agrotóxicos.

Entretanto, a extensão de envenenamento agudo e crônico no Amazonas é difícil de ser estimada. O Centro para Informaçôes Toxicológica do Hospital Universitário registrou 68 casos de intoxicação entre 1995 e 2000 (Waichman et al, 2002), a maioria deles acidentes domésticos e tentativas de suicídio (exposição intencional), porém com escassos registros da contaminação ocupacional. O número de casos derivados da exposição ocupacional é desconhecido, pois a maioria deles não é registrada devido a que acontecem nas áreas rurais longe dos serviços de saúde. No caso de envenenamento crônico, são mal diagnosticados porque raramente os agrotóxicos são identificados como agentes causadores dos sintomas, principalmente quando a diagnose é baseada unicamente nos sintomas apresentados pelo paciente. Os serviços de saúde dos municípios do interior do Estado do Amazonas sempre atuaram em condições precárias frente à realidade do estado de saúde da população local e os riscos impostos pelas condiçôes ambientais da região.

Além da exposição ocupacional dos agricultores, suas famílias, e dos consumidores por meio do consumo de frutas e hortaliças com resíduos de agrotóxicos, é possível que esteja acontecendo a contaminação dos peixes através da contaminação da água, o que representa um risco adicional para as populaçóes locais e para os consumidores de outros locais, principalmente se considerarmos que o peixe é a 
principal fonte de proteínas para a população do Estado do Amazonas (Batista, 1998 ).

Para determinar o grau atual de contaminação e o risco do uso dos agrotóxicos tanto para as populaçôes humanas quanto para o ambiente é necessária a realização da uma avaliação integrada de risco.

\section{A PROPOSTA DE AVALIAÇÃO INTEGRADA DE RISCO}

O objetivo do estudo que está sendo inicialmente conduzido nos municípios de Manaus, Iranduba, Careiro da Várzea e Manacapuru, no Estado do Amazonas, Brasil (Figura 1) é realizar uma Avaliação Integrada de Risco que permita a implantação de um sistema de controle e monitoramento do uso de agrotóxicos no estado do Amazonas.

O objetivo da avaliação de risco é fornecer suporte para a tomada de decisões estimando os riscos de efeitos adversos para a saúde humana e o ambiente de diversos fatores ambientais estressores (Sutter II et al., 2003; Sekizawa et al., 2003; Ross \& Birnbaum, 2003). Entretanto, por razões práticas as ferramentas e os métodos de avaliação internacionalmente utilizados para avaliar riscos para a saúde humana têm sido desenvolvidos independentemente daqueles utilizados para a avaliação de riscos ambientais (Van Leeuwen \& Hermens, 1995; Munns et al., 2003, Sutter II et al., 2005; Sekizawa \& Tanabe, 2005). Assim, com o crescente reconhecimento da necessidade de uma proteção mais efetiva das pessoas e do ambiente, atualmente está se propondo uma abordagem integrada da avaliação de risco (Sutter II et al., 2003).

A Avaliação Integrada de Riscos se constitui numa abordagem científica que combina o processo de estimação de riscos para o homem, a biota e os recursos naturais em uma única avaliação (Sutter II et al., 2003, Sutter II et al., 2005).
Desta forma, a proposta que está sendo aqui apresentada tem caráter inovador, uma vez que pretende realizar uma avaliação humana e ambiental integrada do uso de agrotóxicos, a partir de uma abordagem holística que permita uma maior eficácia no processo de manejo de riscos e na tomada de decisóes para a proteção humana e ambiental.

\section{AS ETAPAS DA AVALIAÇÃO INTEGRADA DE RISCOS DO USO DE AGROTÓXICOS NO ESTADO DO AMAZONAS}

A avaliação de risco consiste em três etapas ou fases (Figura 2). A primeira etapa é o delineamento ou formulação do problema que foi apresentada, de forma resumida, no início deste trabalho. A formulação do problema permite realizar a avaliação da exposição. Esta fase do processo de avaliação integrada de risco tem como objetivos determinar as concentraçôes dos agrotóxicos nos compartimentos bióticos e abióticos do ecossistema, melhorar o entendimento dos processos ou fatores que determinam a exposição e identificar os níveis de estresse do ambiente em relação aos agrotóxicos (Römbke \& Moltmann, 1996).

Para caracterizar a exposição, além de conhecer os pesticidas que são utilizados é necessário conhecer as quantidades utilizadas, a freqüência e os locais de utilização. No estado do Amazonas, esta fase já foi concluída, e, portanto hoje temos conhecimento dos agrotóxicos utilizados. Os agricultores dos municípios de Manaus, Manacapuru, Iranduba e Careiro da Várzea utilizam 18 tipos diferentes de agrotóxicos (11 inseticidas, 4 fungicidas e 3 herbicidas) (Tabela 1) (Waichman et al., 2002, 2007). Os inseticidas são os agrotóxicos mais utilizados, a maior parte deles classificados toxicologicamente como moderadamente a extremamente
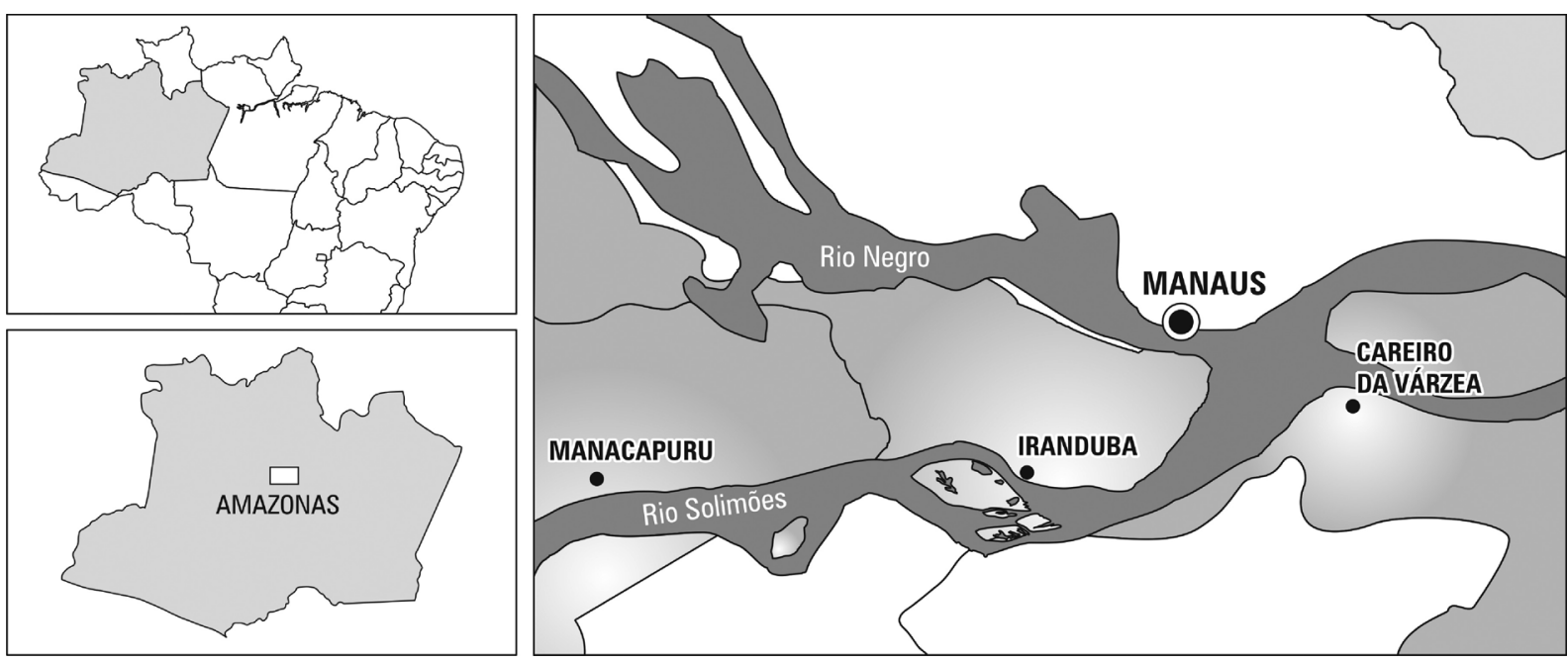

Figura 1 - Localização da área de implantação da Avaliação Integrada de Risco do Uso de agrotóxicos. 


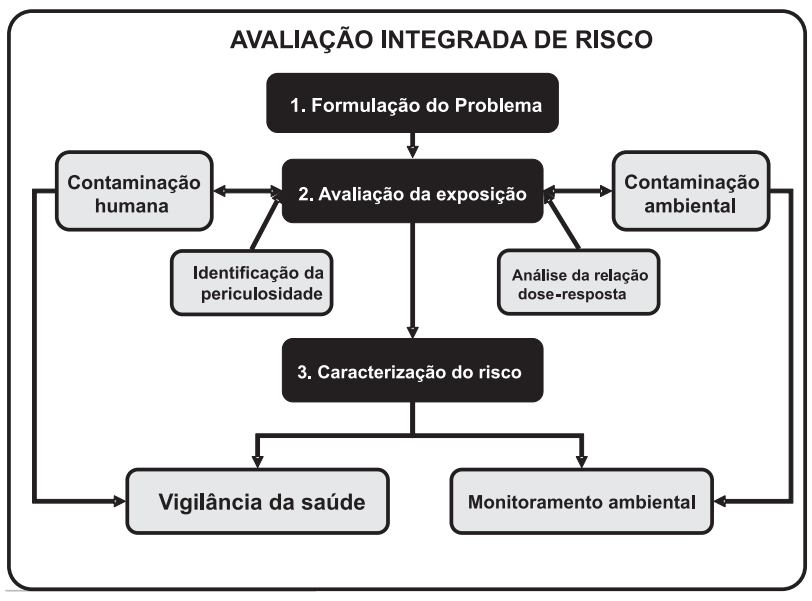

Figura 2 - Esquema com as fases do processo de Avaliação Integrada de Risco

Tabela 1 - Frequência de uso dos diferentes agrotóxicos na área de estudo e classificação toxicológica e ecotoxicológica no Brasil.

\begin{tabular}{lll}
\hline Ingrediente ativo & Classificação toxicológica & \% de uso* \\
\hline Inseticidas & III & 1,3 \\
\hline Clorpirifós & IV & 64,5 \\
\hline Deltametrina & II & 1,3 \\
\hline Endosulfan & III & 18,5 \\
\hline Malationa & II & 13,5 \\
\hline Metamidofós & I & 42,2 \\
\hline Parationa-metilica & II & 1,3 \\
\hline Triclorfom & & \\
\hline Fungicidas & IV & 1,3 \\
\hline Benomyl & IV & 38,2 \\
\hline Oxicloreto de cobre & IV & 25,1 \\
\hline Mancozeb & IV & 5,3 \\
\hline Herbicidas & & \\
\hline Ácido 2,4-dichlor. & II & \\
\hline Glifosato & IV & \\
\hline
\end{tabular}

* \% de uso baseada em respostas múltipas uma vez que os agricultores utilizam vários agrotóxicos.

I. Extremamente tóxico; II. Altamente tóxico; III. Moderadamente tóxico; IV. Levemente tóxico

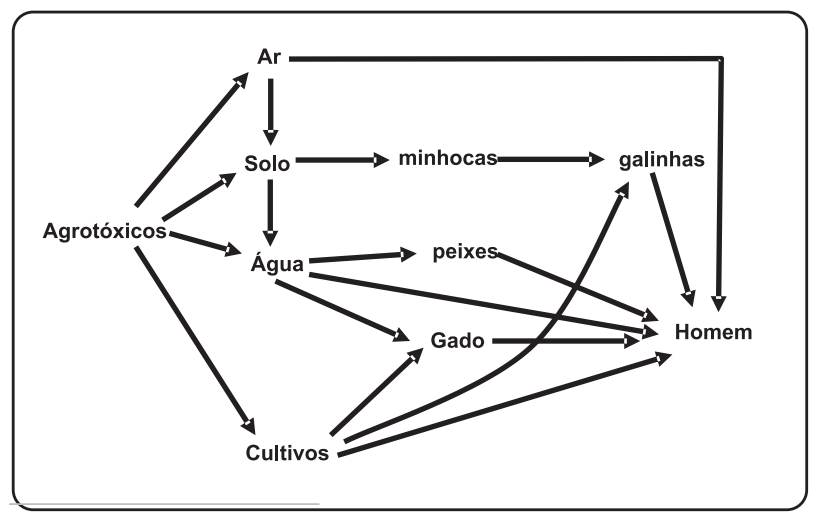

Figura 3 - Representação esquemática das possíveis vias de exposição ambiental e humana

tóxicos (Ministério da Saúde, 1984). O uso de herbicidas está aumentando devido à vantagem econômica representada pelo baixo custo de sua utilização quando comparado ao custo da mão de obra necessária para a limpeza das áreas de plantio (Waichman et al., 2002).

Para a avaliação da exposição é necessário o estabelecimento do modelo conceitual que representa as fontes de agrotóxicos no ambiente e as vias de exposição, unindo a fonte aos receptores humanos e ambientais (Figura 3). Como fontes de agrotóxicos para o ambiente no Amazonas temos os processos de preparação e mistura, aplicação por borrifação, o processo de descarte das sobras de agrotóxicos, da lavagem dos equipamentos e roupas utilizadas durante a aplicação e do descarte das embalagens.

Os diferentes organismos e o homem podem estar expostos diretamente aos agrotóxicos durante sua aplicação (borrifação). Nesta via de exposição também é incluída a exposição da água e do solo por meio da dispersão pelo vento do spray gerado durante a borrifação. No caso do homem, a exposição direta aos agrotóxicos se processa durante a preparação, a mistura, a aplicação e o descarte, pelo contato dérmico ou pela inalação.

Indiretamente, existe um maior número de rotas de exposição. O homem, os animais domésticos e silvestres de forma geral estão expostos pela alimentação com as culturas nas quais foram aplicados os agrotóxicos. Os animais silvestres e domésticos podem se contaminar a partir da água, do solo, e de outros recursos ambientais contaminados nos quais vivem ou dos quais se alimentam. Tanto o homem como os animais domésticos e silvestres podem se contaminar bebendo água contaminada. Estas rotas de exposição e a quantificação da exposição são avaliadas a partir da determinação dos níveis de agrotóxicos nos diferentes compartimentos do ecossistema e nos seres humanos. No nosso caso, primeiramente as concentrações de agrotóxicos serão determinadas na água, 
no solo, nos peixes, em organismos do solo (minhocas), nas frutas e verduras comercializadas e no homem.

Além da caracterização da exposição, um elemento fundamental na avaliação da exposição é a determinação ou caracterização dos efeitos dos agrotóxicos nos seres humanos e demais organismos vivos. Este passo da avaliação de risco consiste em duas fases distintas: 1) a identificação da periculosidade, onde dados da literatura são compilados de forma a determinar se existe a chance do agrotóxico entrar no ecossistema e em quais compartimentos, podendo causar danos tanto nos seres humanos quanto no ambiente e 2) a análise da relação dose-resposta a partir de dados da literatura sobre testes em diferentes organismos.

A análise da relação dose-resposta é uma das etapas mais críticas na avaliação de risco, principalmente no Estado do Amazonas, pois não temos dados da relação dose-resposta para os organismos da região, pois a maior parte dos testes de laboratório com este propósito foi desenvolvida com organismos das regiôes temperadas.

Portanto, como parte da avaliação de risco, serão realizados testes com diversos organismos terrestres e aquáticos com a finalidade de estabelecer os principais parâmetros para avaliação da dose-resposta:

Concentração de efeito não observado (CENO): Maior concentração nominal da amostra que não causa efeito deletério estatisticamente significativo na sobrevivência dos organismos, nas condições de ensaio.

Concentração de efeito observado (CEO): Menor concentração nominal da amostra que causa efeito deletério estatisticamente significativo na sobrevivência dos organismos, nas condiçõos de ensaio.

Concentração letal mediana $\left(\mathrm{CL}_{50}\right)$ : Concentração real da amostra que causa efeito agudo a $50 \%$ dos organismos no tempo de exposição, nas condiçôes de ensaio.

Concentração efetiva zero $\left(\mathrm{CE}_{\text {zero }}\right)$ : Maior concentração da amostra onde não se observa efeito agudo aos organismos no tempo de exposição, nas condiçôes de ensaio.

A terceira e última etapa é denominada de caracterização de risco e tem por objetivo avaliar a magnitude dos riscos para a saúde humana e o ambiente. Como resultados deste processo são avaliados tantos os aspectos qualitativos quanto quantitativos do risco. Esta etapa em geral é realizada de forma progressiva, em quatro níveis, sendo que em cada nível é avaliado o risco, tomando como base as informaçōes sobre exposição, a toxicidade do agrotóxico e os efeitos toxicológicos, de forma a determinar a probabilidade de efeitos adversos para o ambiente e o homem (Figura 4). Se a avaliação aponta riscos no nível anterior então um refinamento da avaliação é realizado no nível seguinte.

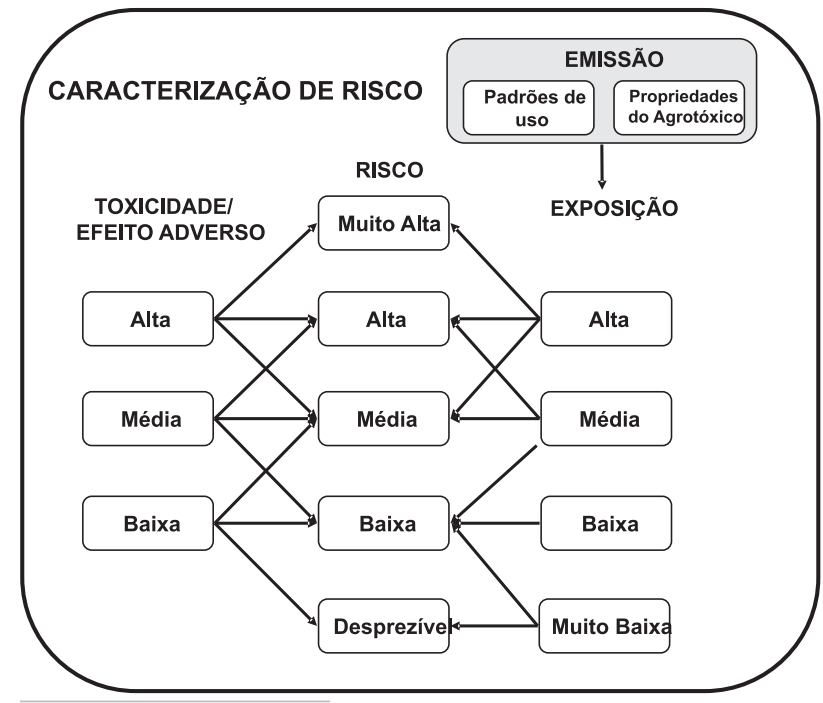

Figura 4 - Representação esquemática do processo de caracterização do risco

Assim, para cada agrotóxico avaliado são comparadas as concentrações estimadas no ambiente (CAE) com as concentrações de efeitos agudos $\left(\mathrm{CL}_{50}\right)$ e crônicos $(\mathrm{CENO})$ a partir do cálculo do Quociente de Risco (QR). Estes valores são comparados com níveis críticos de risco, que variam para os diferentes compartimentos ambientais analisados (e.g. o nível crítico para risco agudo alto em ambiente aquático é $0,5)$. Se o quociente de risco é menor que o nível crítico o agrotóxico não apresenta risco para a o ambiente e o homem. Entretanto, se o quociente de risco é maior que o valor crítico estabelecido existe risco para a população e o ambiente e uma avaliação mais refinada deve ser feita. Desta forma poderemos determinar quais são os agrotóxicos que se apresentam como mais problemáticos no Estado e apresentam maiores riscos, e a partir disso, poderá ser estabelecido o manejo do risco.

Resumidamente, a avaliação de risco nos permitirá achar as respostas para as seguintes questōes:

Quais são os principais fatores que contribuem para o risco?

Quais são os efeitos adversos dos agrotóxicos?

Quais são as vias de exposição nas condições de uso realizadas pelos agricultores?

Quais é o nível aceitável de risco?

\section{O MANEJO DOS RISCOS}

O manejo de riscos é uma etapa posterior à avaliação onde são determinadas as medidas que deverão ser tomadas para a redução do risco. Para isto, além de se considerar os efeitos adversos estimados na avaliação de risco, devem ser consideradas as implicações socioeconômicas e políticas de 
medidas de redução do uso e exposição de agrotóxicos ou opçôes alternativas ao seu uso.

A partir das respostas encontradas para as questōes acima apresentadas, três estratégias básicas serão consideradas para o manejo dos riscos: 1) o uso correto e adequado dos agrotóxicos; 2) a redução do uso dos agrotóxicos e 3) a introdução de práticas alternativas como o Manejo Integrado de Pragas.

Para isso, serão delineados e implantados programas específicos de educação, treinamento e informação dos agricultores e também dos extensionistas, com o objetivo de ajudar o público alvo a entender os riscos dos pesticidas para a saúde, a necessidade do uso de equipamentos de proteção individual, práticas adequadas de higiene e descarte das embalagens, reconhecimento rápido dos sintomas de intoxicação ações para o pronto socorro das pessoas intoxicadas, e desta forma induzir a adoção de práticas de uso mais seguro que diminuam o risco de exposição humana e ambiental e a criação de uma nova consciência entre os agricultores, permitindo a construção de uma agricultura ambientalmente sustentável no estado do Amazonas.

\section{BIBLIOGRAFIA CITADA}

Ecobichon, D. J. 2001.Pesticide use in developing countries. Toxicology, 160: 27-33.

IBGE (Instituto Brasileiro de Geografia e Estatística) 1988. Censo Agropecuário 1985-1986.

IBGE (Instituto Brasileiro de Geografia e Estatística). 1998. Censo Agropecuário 1995-1996.

Munns, W.R.; Kroes, R.; Veith, G.; Sutter II, G.W.; Damstra, T.; Waters, M.D. 2003. Approaches for integrates Risk Assessment. Hum. Ecol. Risk Asses., 9(1): 267-272.

Nina, N.C.S. 2002. Caracterização sócio-econômica-ambiental do uso de agrotóxicos em uma área de várzea do município de Manacapuru - Am (Amazônia Central). Dissertação de Mestrado, Programa de Pós-Graduação em Ciências do Ambiente e Sustentabilidade na Amazônia. Universidade Federal do Amazonas. 123pp.
Noda, S.N.; Pereira, H.S.; Branco, F.M.C.; Noda, H. 1997. O trabalho nos sistemas de produção de agriculturas familiares na várzea no estado do Amazonas. In: Noda, H; Souza, L.A.H.; Fonseca, O.J.M. (Eds.), Duas décadas de contribuição do INPA à pesquisa agronômica no trópico úmido. INPA. página

Römbke J.; Moltmann, J. 1996. Applied Ecotoxicology. CRC Press, Boca Raton, FL, USA. 282pp.

Roos P.S.; Birnbaum L.S. 2003. Integrated Human and Ecological Risk Assessment: A case study of persistent pollutants (POPs) in human and wildlife. Hum. Ecol. Risk Asses., 9(1): 303-324.

Sekizawa J.; Suter II, G.; Birnbaum, L.S. 2003. Integrated Human and Ecological Risk Assessment: A case study of tributyltin and tyiphenyltin compunds. Hum. Ecol. Risk Asses., 9(1): 325-342.

Sekizawa, J.; Tanabe S. 2005. A comparison between integrates risk assessment and classical health/environment assessment: emerging beneficial properties. Toxicology and Applied Pharmacology, 207: S617-S622.

Sutter II, G. W.; Vermeire, T.; Munns, W.R.; Sekizawa, J. 2003. Framework for the intergration of health and ecological risk assessment. Hum. Ecol. Risk Asses., 9(1): 281-301.

Sutter II, G.W.; Vermeire, T.; Munns, W.R.; Sekizawa, J. 2005. An integrated framework for health and ecological risk assessment. Toxicology and Applied Pharmacology, 207: S611-S616.

Van Leeuwen C.J.; Hermens, J.L. M. 1995. Risk assessment of chemicals: An introduction. Kluwer Acad Publ, Dordrecht, 374pp.

Waichman, A.V.; Römbke, J.; Ribeiro, M.O.A.; Nina, N.C.S. 2002. Pesticide use in the Amazon State, Brazil. Environ. Sci. Pollut. Res., 9(6):423-428.

Waichman, A. V.; Römbke, J.; Nina, N.C.S. 2003. Agrotóxicos: elemento novo na Amazônia. Ciência Hoje, 32(190): 70-73.

Waichman, A.V.; Eve, E.; Nina, N.C.S. 2007. Do farmers understand the information displayed on pesticide product labels? A key question to reduce pesticides exposure and risk of poisoning in the Brazilian Amazon. Crop Protection, 26(4): 576-583

Recebido em 28/09/2004

Aceito em 27/12/2007 